Revue internationale P.M.E.

Économie et gestion de la petite et moyenne entreprise

\title{
Les relations entre constructeurs et fournisseurs : l'exemple de l'industrie automobile
}

\section{Bernard Guilhon}

Volume 6, numéro 1, 1993

URI : https://id.erudit.org/iderudit/1008165ar

DOI : https://doi.org/10.7202/1008165ar

Aller au sommaire du numéro

Éditeur(s)

Presses de l’Université du Québec

ISSN

0776-5436 (imprimé)

1918-9699 (numérique)

Découvrir la revue

Citer cette note

Guilhon, B. (1993). Les relations entre constructeurs et fournisseurs : l'exemple de l'industrie automobile. Revue internationale P.M.E., 6(1), 87-99.

https://doi.org/10.7202/1008165ar
Résumé de l'article

Cet article a pour but d'analyser le mouvement de désintégration verticale et ses répercussions sur les fournisseurs et sur les formes de coordination et de coopération établies avec les constructeurs. Les modes de fonctionnement des firmes européennes et japonaises présentent des différences assez marquées. 


\title{
Notes de recherche
}

\section{Les relations entre constructeurs et fournisseurs: l'exemple de l'industrie automobile}

Bernard GUILHON* Université d'Aix-Marseille II

\begin{abstract}
RÉSUMÉ
Cet article a pour but d'analyser le mouvement de désintégration verticale et ses répercussions sur les fournisseurs et sur les formes de coordination et de coopération établies avec les constructeurs. Les modes de fonctionnement des firmes européennes et japonaises présentent des différences assez marquées.
\end{abstract}

\section{ABSTRACT}

The purpose of this article is to analyse the trend of vertical disintegration and its consequences for the suppliers and for the forms of conjonction and cooperation with the automakers. The mode of operation of European and Japanese firms are well-opposite.

\section{RESUMEN}

La finalidàd de este articulo es analizar el movimiento de desintegracion vertical y sus repercusiones sobre los provedores y sobre las formas de coordinaciones y de cooperaciones establecidas con los constructores. Los modos de funcionamento de los firmas europeas y japoneses presentan diferencias bastante acentuadas.

* Bernard Guilhon est professeur de la Faculté de sciences économiques. Il est le responsable du département d'économie industrielle du C.E.F.I. qui est une unité associée au CNRS. Adresse : Château Lafarge, 13290 Les Milles, France. 


\section{Introduction}

Le but de cet article est de s'interroger sur l'émergence de nouveaux modes organisationnels qui apparaissent au début des années 80 dans l'industrie automobile européenne. Les refontes organisationnelles sont synonymes à la fois de transformations internes aux firmes et de transformations externes qui affectent les relations et les formes de collaboration entre fournisseurs - et notamment les P.M.E. - et les constructeurs.

Les nouveaux principes d'organisation s'établissent progressivement dans une activité dont le caractère mature ${ }^{1}$ peut être mis en évidence à partir de deux critères principaux mais non exclusifs : le taux de croissance rétrospectif et prospectif de la demande et l'intensité de la pression concurrentielle appréciée par les modifications des parts de marché des firmes en France (cf. annexe 1).

Les modifications constatées vont dans le sens d'une désintégration verticale des firmes qui peut être analysée sur le plan théorique à partir des approches macro-économiques de la spécialisation flexible, du post-fordisme ou encore de la thèse schumpétérienne des «longues vagues » de développement économique (Amin et Dietrich, 1991). L'approche théorique centrée sur les firmes met l'accent soit sur la notion de coûts de transaction pour justifier l'existence de formes intermédiaires entre le marché et la hiérarchie (Williamson, 1991), soit sur la multiplicité d'arrangements organisationnels (sous-traitance, franchise, alliance stratégique, ...) attribuable à des contextes différenciés en termes de marchés, de technologies et de savoir-faire, d'environnements socioculturels, etc. (Granovetter, 1985 ; Powell, 1990).

La présente contribution retient une autre démarche : elle part de constats résultant des enquêtes et des observations effectuées dans un certain nombre de pays, ces constats nous permettront de dégager, dans une seconde étape, le contenu des relations productives entre les firmes. Dans un troisième temps, nous insisterons sur les particularités des firmes européennes et des firmes japonaises.

1. Les chiffres et les courbes présentés dans l'annexe 1 établissent le caractère mature de l'industrie. Cela ne signifie pas que le produit automobile soit lui-même mature, c'est-à-dire saturé sur le plan technologique. 


\section{Enquêtes et observations}

\subsection{Les statistiques à notre disposition expriment, avec des décalages, la tendance à la déverticalisation des grandes firmes de construction automobile}

$\left.\begin{array}{lll}\multicolumn{3}{c}{\begin{array}{c}\text { TABLEAU 1 } \\ \text { La désintégration verticale des firmes allemand } \\ \text { (valeur ajoutée/chiffre d'affaires en }\end{array}} \\ \hline \multicolumn{2}{c}{\mathbf{1 9 7 8}}\end{array}\right)$

Source: IG Metall. Chiffres cités par B. DANKBAAR: «Problems and prospects of the west german automotive components industry ». Colloque de Grenoble, 1989.

En ce qui concerne les principales firmes japonaises, le pourcentage de composants fabriqués en interne s'établit à $35 \%$ au début des années 60 et à $25 \%$ à la fin des années 80 , alors qu'il avoisine $52 \%$ pour les firmes américaines au milieu des années 80 (Mitsubishi Research Institute, 1987).

Les prévisions établies pour la décennie 90 indiquent que les constructeurs européens feront fabriquer par les fournisseurs $90 \%$ des équipements électroniques d'un véhicule, 30 à $40 \%$ des systèmes de suspension, $70 \%$ des dispositifs électriques, etc. (Booz, Allen et Hamilton, 1987).

\subsection{Comment expliquer cette tendance ?}

Plusieurs raisons ont été avancées parmi lesquelles on relève (Dankbaar, 1989) :

- Le manque de place dans les usines d'assemblage, les nouvelles technologies exigeant plus de mètres carrés par véhicule fabriqué, ce qui conduit les constructeurs à se débarrasser des activités dites « secondaires » et, de ce fait, à réduire les dépenses en capital (installations et équipements).

- La faiblesse relative des coûts de production des fournisseurs, imputable en partie au différentiel de coût salarial. 
- Les coûts de stockage des pièces détachées et des produits intermédiaires. Il est vrai que l'adoption de procédures comme le juste-à-temps tend à limiter la croissance de ces coûts de fonctionnement.

- Les évolutions technologiques à l'œuvre dans les domaines de l'électronique et des nouveaux matériaux ont incité les constructeurs à tirer parti du capital de connaissances accumulé par les fournisseurs qui ont sensiblement accru leurs dépenses de $\mathrm{R}$ et $\mathrm{D}$ au cours des quinze dernières années : ce qui est une façon de mutualiser les coûts et les risques entre plusieurs intervenants (Guilhon, 1992).

- La désintégration verticale n'est pas seulement une tendance : elle peut être utilisée comme une menace pour exercer des pressions sur les activités de production interne de façon à améliorer les performances de productivité et de qualité et à persuader les salariés d'accepter des modifications d'horaires de travail.

\subsection{Quelles sont les répercussions de ce mouvement sur les différentes catégories de fournisseurs?}

Les fournisseurs représentent une catégorie hétérogène formée de firmes qui se différencient par leur taille, leurs compétences technologiques et leur environnement concurrentiel (Doleschal, 1989 ; Enrietti, 1989) :

1. Au sommet de la pyramide, on trouve deux catégories de firmes :

- celles pour lesquelles le marché automobile ne représente qu'une faible part de la production (Siemens, par exemple), mais dont le savoir-faire technologique favorise la croissance de leur part de marché, que ce soit par diversification ou expansion d'activités déjà existantes ;

- celles qui représentent les fournisseurs spécialisés dans les équipements complexes. Il peut s'agir là de grandes firmes ou de P.M.E. (Bosch, Valeo, etc.). Les entreprises appartenant à ce groupe se distinguent par leur capacité de développement d'un produit, les investissements élevés en $\mathrm{R}$ et $\mathrm{D}$, le niveau de connaissances technologiques incorporées dans le produit et l'aptitude à fournir de vastes marchés d'utilisateurs. La désintégration verticale conduit à reconnaître à ces fournisseurs de premier rang une responsabilité directe en matière de coût, qualité et fiabilité du produit.

2. Les fournisseurs de second rang (en général des P.M.E.) ont des compétences technologiques plus limitées (composants simples ou peu complexes). La désintégration verticale est synonyme de pression sur les coûts et 
les prix, de qualité accrue et de raccourcissement des délais. C'est ce qu'indique le P.-D.G. d'une entreprise spécialisée dans la visserie-boulonnerie et les pièces de fixation (Raymond, 1989). Les contraintes logistiques exprimées par le flux tendu conduisent à fixer des délais de 48 heures entre la commande et la livraison des pièces sur le site d'installation, avec un stock de sécurité compris entre 0 et 24 heures Pour conserver ses marchés, la firme doit investir dans des technologies de production et des systèmes télématiques qui la connectent aux donneurs d'ordre de façon à traiter les flux d'information qui lui parviennent quotidiennement. L'un des risques réside dans la possibilité d'une inadéquation des commandes avec les cadences prévisionnelles. Or, "plus les délais sont courts, plus la possibilité de réagir se réduit » (p. 25).

3. Les fournisseurs de produits de bas de gamme (pièces en plastique, par exemple) soumis à de fortes pressions de coût représentent les sous-traitants de capacité. La désintégration verticale ne change pas fondamentalement leur statut par rapport aux formes de sous-traitance de volume de la période fordiste.

\section{L'analyse des relations productives}

Le processus de réorganisation des structures productives modifie le nombre d'entreprises qui interviennent dans la production d'un véhicule et le contenu des normes de production.

\subsection{La redéfinition des rapports}

Sur le plan quantitatif, la redéfinition des rapports prend la forme d'un processus de sélection qui conduit les constructeurs à réduire le nombre global de leurs fournisseurs qui sont intégrés dans le cycle global de fabrication en se basant sur une série de critères tels que la capacité de conception et l'étendue des compétences technologiques, le montant des ventes, le niveau de compétitivité, etc. C'est ce qui a permis à Peugeot S.A. de réduire le nombre de ses fournisseurs de 1750 en 1984 à 950 en 1989. Chez Fiat, les commandes libérées par la disparition de 350 entreprises ont été transférées à des fournisseurs qui satisfaisaient aux critères indiqués, ce qui a entraîné des baisses de coût évaluées à $2 \%$ et plus (Enrietti, $1989: 138$ ).

\subsection{La coordination des flux et la coopération des unités}

La définition de nouvelles normes de production accompagne le mouvement d'externalisation d'activités dont la logique se situe à la fois sur le plan de la coordination des flux et de la coopération des unités. 
1. La coordination pose directement le problème de l'articulation des flux de produits et d'informations entre fournisseurs et constructeurs. L'introduction des technologies d'information et de communication (T.I. \& C.) - c'est-àdire des technologies qui ont un contenu organisationnel (ordinateurs, systèmes communicationnels informatisés, télématique, etc.) - accroît l'intégration informationnelle des fournisseurs par rapport à un centre de décisions. Le développement de procédures organisationnelles comme la multiplication des échanges de données inter-sites et inter-unités, l'intégration gestionnaire logistique avec traitement informatisé des flux de commandes depuis les clients jusque chez les fournisseurs (juste-à-temps), la normalisation des modes de communication, etc., a pour but d'optimiser essentiellement des paramètres techniques : délais, coûts, niveau de stock, taux de rebut.

En particulier, l'implantation des systèmes télématiques sous l'impulsion des constructeurs a eu pour effet d'abaisser les délais de livraison, mais aussi les délais de conception et de lancement d'un nouveau véhicule. La diffusion de la C.A.O. aux fournisseurs et la normalisation des fichiers qui l'accompagne permettent notamment aux concepteurs de composants et aux fabricants d'équipements de production et de systèmes de mesure spécialisés de «pouvoir accéder à des bases de données dans lesquelles sont tenues à jour toutes les informations relatives au véhicule » (Brousseau, 1991). Précisons que le délai de conception et de lancement est estimé à 4 ans au Japon et à $7 / 8$ ans en Europe et aux U.S.A. (Mitsubishi Research Institute) ${ }^{2}$.

Les systèmes télématiques inter-entreprises peuvent également favoriser le développement de normes générales ${ }^{3}$ qui ont pour but de rationaliser les échanges d'informations. Telle est la signification du projet O.D.E.T.T.E. ${ }^{4}$ qui a pour objectif de promouvoir l'adoption de standards techniques communs pour l'échange électronique de données entre constructeurs et fournisseurs en Europe.

En ce qui concerne les coûts, la diffusion de la C.A.O. a eu pour résultat de réduire le nombre de pièces de carrosserie à assembler et d'accroître la

2. General Motors s'est lancé dans un vaste plan de compression des délais de conception et de lancement d'un véhicule, l'objectif étant de parvenir à 18-20 mois en 1995.

3. Les constructeurs peuvent agir de deux façons : en instituant des normes «privatives», ils rehaussent les barrières à la mobilité et accroissent les coûts de sortie du système pour les fournisseurs étroitement dépendants du marché automobile. À l'inverse, la normalisation des données sur le plan européen (normes dites générales) augmente pour les fournisseurs les possibilités de sortie de la coalition, voir à ce sujet Brousseau, 1991.

4. O.D.E.T.T.E. Organization for Data Exchange through Teletransmission in Europe. 
standardisation des composants ${ }^{5}$.C'est la raison pour laquelle il peut paraître simplificateur de rattacher la création de ces nouveaux modes organisationnels à la recherche d'un seul objectif qui serait la flexibilité. Il y a bien flexibilité accrue du système d'entreprises ainsi constitué (par la réduction de la taille des lots, la multiplication des modèles et des variantes, l'adoption de procédures qui permettent un haut degré d'adaptation,etc.), mais un grand nombre de fournisseurs deviennent plus spécialisés dans la production d'un composant ou d'un sous-système, ce qui renforce l'apparition d'économie d'échelle. C'est donc à la fois la recherche de flexibilité et de normalisation qui motive la création de ces formes d'organisation.

2. La coopération permet de préciser comment se nouent les rapports économiques entre les firmes. Les principes de contractualisation qui soustendent ces organisations (Guilhon, 1992) interviennent dans les domaines suivants :

- Les contrats de fourniture sont établis sur une période suffisamment longue ( 4 ans en général) pour permettre à un fournisseur d'amortir les investissements réalisés.

- Les contrats de développement d'un nouveau modèle incitent les fournisseurs à investir et à engager des dépenses de recherche, dans le cadre des directions définies par le constructeur.

- Les modifications de prix tiennent le plus grand compte de l'évolution des coûts de production et des gains de productivité des fournisseurs. Les prix sont négociés et les négociations sont conduites dans l'intérêt des constructeurs et des fournisseurs.

- Des transferts de connaissances s'effectuent entre les firmes à différents niveaux de la structure afin d'impulser une dynamique collective d'apprentissage technologique.

Voilà qui conduit à donner les caractéristiques qui distinguent les firmes européennes des firmes japonaises.

\section{Homogénéisation des principes, différenciation des pratiques}

Quels sont les traits caractéristiques du processus de restructuration qui affecte l'industrie automobile?

5. «On est passé de 18 modèles base des marques Fiat et Lancia construits sur 11 types différents de châssis au début des années 80 , à 14 modèles base construits sur seulement 5 châssis différents, un nombre qui correspond aux principales catégories du marché. » (Enrietti, 1989 : 139). 


\subsection{L'influence du modèle japonais}

Le nouveau mode d'organisation trouve ses racines au Japon, pays caractérisé dans les années 50 par un marché intérieur « ridiculement étroit, [qui] était de surcroît encombré par une quantité invraisemblable d'offreurs et de modèles [...]. Dans ces conditions, la concurrence et la compétitivité se déterminaient sur la capacité à satisfaire rapidement des demandes courtes et variées (Coriat, 1991). Ce système adapté à la production en séries limitées de produits différenciés et variés repose sur un certain nombre de principes internes (rehaussement des qualifications ouvrières, juste-à-temps) et relationnels (procédures contractuelles entre firmes). Ces principes généraux d'organisation et de fonctionnement caractérisent ce que nous avons appelé une firme-réseau qui est un mode d'organisation grâce auquel se constituent, se spécifient et s'allouent des ressources mobilisées en vue de réaliser un projet productif (Guilhon, 1992). Les firmes japonaises adoptent ce mode d'organisation en désintégrant des activités qu'elles assumaient auparavant, mais surtout en multipliant les filiales (taux de contrôle $\geq 50 \%$ ) et les firmes affiliées (taux de contrôle $\geq 20 \%$ ) avec lesquelles elles tissent des relations contractuelles comme avec les firmes indépendantes (Aoki, 1984).

De nombreux auteurs reconnaissent l'influence exercée par le «modèle japonais » sur les firmes européennes et américaines, incapables de concilier adaptation à la demande et organisation fordienne de la production (Womack, Jones et Ross, 1991 ; Gianfaldoni, 1992). Ces principes, expérimentés avec succès au Japon, vont se diffuser en Europe, plus lentement aux USA, sous l'influence notamment des missions d'étude effectuées au Japon, sous l'influence également des investissements directs réalisés par les firmes japonaises à l'étranger. Ces principes vont se concrétiser en Europe en permettant aux particularités nationales de s'exprimer, notamment en ce qui concerne les relations avec les fournisseurs et les sous-traitants.

\subsection{La quasi-intégration verticale}

La quasi-intégration verticale (Q.I.V.) exprime l'application de ces principes par les firmes européennes. Selon Enrietti (1983), la Q.I.V. établit un système hiérarchique entre fournisseurs de statut différent, dont les relations avec le constructeur s'interprètent en termes de stabilité, de pourcentage du chiffre d'affaires réalisé, d'activités qui ne se limitent pas à la fabrication, mais s'étendent à la recherche et au marketing. La Q.I.V. apparaît ainsi comme le « volet vertical » d'une firme-réseau, puisqu'elle permet à la grande firme, dont les rapports ont été strictement définis avec les entreprises externes, de 
bénéficier des avantages de l'intégration verticale sans en avoir les inconvénients (Leborgne, 1987).

\subsection{Les différences}

Des différences apparaissent cependant entre firmes européennes et japonaises. Nous insisterons plus particulièrement sur deux d'entre elles :

1. Si les fournisseurs de premier rang occupent dans les deux cas une place privilégiée, leurs relations avec les constructeurs sont plus formalisées au Japon. Les groupes japonais organisent en effet leurs rapports à l'intérieur d'un « kyoryokukaï » qui est une association coopérative constituée avec les fournisseurs dans le but de faciliter les échanges d'informations et de rechercher des solutions en commun (Mitsubishi Research Institute, p. 4). Ce système s'accompagne de « contrats d'assemblage » qui consistent à déléguer à des fournisseurs la responsabilité de la fabrication complète d'un sous-système ou d'un composant, lesquels coordonnent à leur tour l'activité de fournisseurs situés à un échelon inférieur (Nishigushi, 1987).

2. Ce système d'organisation « en cascade » se double, selon cet auteur, d'une forme de « contrôle en grappe » qui se différencie nettement des pratiques européennes.

En effet, les relations entre constructeurs et fournisseurs de premier rang en Europe peuvent être décrites sur le plan technologique en termes de dépendance mutuelle et de partenariat. Ce sont des préoccupations technologiques qui sous-tendent ces rapports. Cela ne s'oppose pas à l'élaboration de stratégies propres aux fournisseurs qui internationalisent leurs activités en vue d'étendre leurs marchés (Bochum et Meissner, 1989).

La dépendance par rapport aux constructeurs est plus accentuée au Japon. Un « kyoryokukaï » représente une forme d'organisation sociale grâce à laquelle le constructeur « orchestre » l'interaction sociale des unités sélectionnées : « la première fonction d'un « kyoryokukaï » est de contrôler les fournisseurs et les sous-traitants » et la relation partenariale n'est qu'une « façade » (Nishigushi, $1987: 13-14)$.

Le contrôle sur les firmes situées aux étages inférieurs de la structure s'exerce via les fournisseurs de premier rang. Ceux-ci organisent à leur tour leurs propres fournisseurs et sous-traitants sous la forme d'un « kyoryokukaï » regroupant des firmes à compétence technologique moins élevée, mais avec 
la même finalité : assurer la synchronisation des services et des opérations effectuées et diffuser "la philosophie et les pratiques de fabrication du constructeur » (p. 16). La cohésion de l'ensemble est donc assurée sur le plan de l'organisation par des «agences de contrôle » qui orientent la dynamique collective en harmonisant les pratiques de fonctionnement et en développant des formes d'assistance technique et des programmes de formation destinés aux firmes appartenant aux associations coopératives.

\section{Conclusion}

La désintégration verticale modifie les rapports entre constructeurs et fournisseurs à l'intérieur de constructions organisationnelles baptisées firme-réseau, Q.I.V., etc. Elle s'accompagne de la fixation de nouvelles normes de production que nous avons analysées sur le plan de la coordination des flux et sur le plan de la coopération entre firmes qui reposent sur des principes de contractualisation.

Les configurations étudiées se différencient assez nettement entre firmes japonaises et firmes européennes. Les pratiques de ces dernières semblent privilégier une approche de la technologie conçue comme connaissance (Constant, 1987). Le constructeur cherche à acquérir des actifs externes complémentaires et à tirer parti des savoirs et savoir-faire élaborés par les bases de connaissance et d'expérience des fournisseurs.

En ce qui concerne les firmes japonaises, les rapports s'organisent à l'intérieur de formes plus hiérarchisées dont les dimensions sont à la fois techniques et socio-organisationnelles. La technologie est ici appréhendée comme système complexe, c'est-à-dire comme une articulation de produits, de marchés, de ressources productives, mais aussi de philosophies et de pratiques de fonctionnement. La cohérence organisationnelle suppose l'exercice d'une fonction de contrôle qui peut être déléguée par l'assembleur à des relais qui occupent une position stratégique. 


\section{Bibliographie}

AMIN, A. et M. DiETRICH (1991), «From hierarchy to «Hierarchy», the dynamics of contemporary corporate restructuring in Europe », dans Towards New Europe? Edward Elgar, p. 49-73.

AOKI, M. (1984), «Innovative adaptation through the quasi-tree structure : an emerging aspect of Japanese entrepreneurship », Journal of Economics, 1984, suppl. 4, p. 177-198.

Bochum, U. et H.R. MEISSNER (1989), «The German automotive supply industry : logistics and internationalization », Rencontres Européennes de Grenoble, IREPD-CRESAL, p. 127-136.

Booz, Allen et Hamilton INC. (1987) : «Opportunities for promoting the Dutch automotive supply industry », Ministerie van Economische Zaken, Den Haag.

BROUSSEAU, E. (1991), «Les contrats dans une économie d'échange et de production : technologies de l'information et de la communication et coordination inter-entreprises », Thèse pour le doctorat de sciences économiques, Université Paris-Nord.

CONSTANT, E.W. (1987), « The social locus of technological practice : community, system or organization? »dans The Social Construction of Technological Systems : New Directions in the Sociology and History of Technology., éd. par Bijker, Hughes et Pinch., M.I.T. Press, p. 223-242.

CORIAT, B. (1991), « Penser à l'envers. Travail et organisation dans l'entreprise japonaise », C. Bourgois éditeur.

DANKBAAR, B. (1989), "Problems and prospects of the West German automotive components industry » dans Rencontres Européennes de Grenoble, IREPDCRESAL, sous la direction de E. de Banville et J.J. Chanaron, p. 95-125.

DOLESCHAL, R. (1989), « Just-in-time strategien und betriebliche interessenvertretung in automobile zulieferbetrieben " dans Systemische Rationalisierung und Zulieferindustrie, Campus, Munich, p. 155-205.

ENRIETTI, A. (1989), «La dynamique de l'intégration verticale à la Fiat Auto SPA (1979-1986) », Rencontres Européennes de Grenoble, IREPD-CRESAL, p. $137-149$.

Gianfaldoni, P. (1992), « Réflexions sur le modèle japonais » Papier de Recherche, CEFI, Aix-en-Provence, $30 \mathrm{p}$.

GRANOVETTER, M. (1985), « Economic action and social structure : the problem of embeddedness », American Journal of Sociology, vol. 91, $\mathrm{n}^{\circ}$ 3, p. 481-510.

GuilhoN, B. (1992), « Technologie, organisation et performances : le cas de la firmeréseau », Revue d'Économie Politique, 102 (4), juillet-août, p. 58-87. 
LEBORGNE, D. (1987), « Equipements flexibles et organisation productive », dans Aspects de la Crise, Tome I. Contrat CGP/CEPREMAP, Paris, p. 345-454.

MITSUBISHI RESEARCH INSTITUTE (1987) : «The relationship between Japanese auto and auto parts makers », Automobile Manufacturers Association, Tokyo, 28 p.

NishiguSHI, T. (1987), « Competing systems of automotive components supply : an examination of the Japanese «clustered control» model and the «Alps structure», First Policy Forum International Motor Vehicle Program M.I.T. Niagara-on-theLake, Ontario, Canada, 26 p.

POWELL, W.W. (1990) : « Neither market nor hierarchy : network forms of organization », Research in Organizational Behavior, vol. 12, p. 295-336.

RAYMOND, A. (1989), «L'évolution logistique imposée par le flux tendu à un équipementier automobile et ses limites » dans Rencontres Européennes de Grenoble, IREPD-CRESAL, p. 23-25.

Williamson, O.E. (1991), «Comparative economic organization : the analysis of discrete structural alternatives », Administrative Science Quarterly, p. 269-296.

WoMACK, J.P., D.T. JONES et D. Ross (1991), Le système qui va changer le monde, Dunod, 349 pages. 


\section{ANNEXE 1}

* Taux de croissance annuelle de la demande en Europe occidentale (en \%)

\begin{tabular}{ccc}
\hline $\mathbf{1 9 7 0 - 1 9 8 0}$ & $\mathbf{1 9 8 0 - 1 9 9 0}$ & $\mathbf{1 9 9 0 - 2 0 0 0}$ (prévisions) \\
\hline 3,2 & 1,3 & 1,4 \\
\hline $\begin{array}{l}\text { Source : CSCA, 1990. } \\
\text { * La modification des parts de marché peut être appréciée en considérant l'évolution } \\
\text { du taux de pénétration (en \% de voitures étrangères dans les immatriculations) } \\
\text { des véhicules personnels sur le marché français en 1978 et en 1988. }\end{array}$ \\
\hline Années & $\mathbf{1 9 7 8}$ & $\mathbf{1 9 8 8}$ \\
\hline Taux de pénétration & 22,9 & 38,1 \\
\hline
\end{tabular}

Source : CSCA, 1990.

La pression concurrentielle peut être appréciée également en considérant l'évolution des parts de marché des principales firmes sur le marché français.

Marché France

Évolution des parts de marché

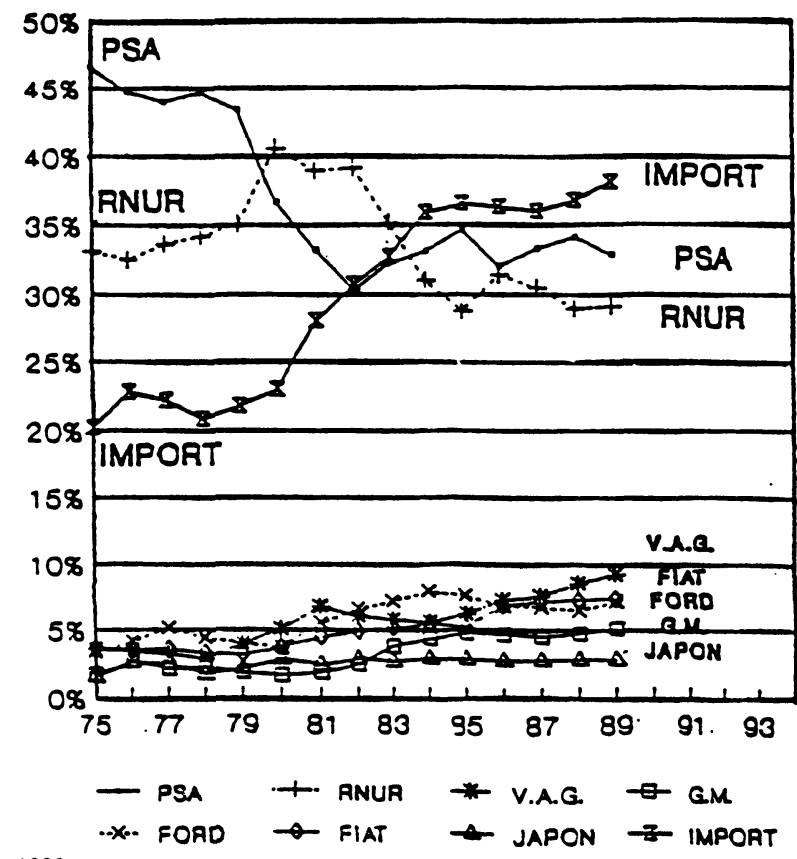

Source : CSCA, 1990. 